\title{
Additive Mixed Sensitivity Design of PID Controllers for Continuous- Time System with Uncertain Time-Delay
}

\author{
TOORAN EMAMI \\ Department of Engineering (Electrical Engineering Program) \\ U. S. Coast Guard Academy \\ 27 Mohegan Ave, New London, Connecticut 06320 \\ UNITED STATES OF AMERICA
}

\begin{abstract}
This paper presents an algorithm for all achievable coefficients of Proportional Integral Derivative (PID) controllers in an integral-derivative plane that stabilizes and satisfies additive mixed sensitivity constraint with an uncertain time delay for a continuous-time system. This algorithm solves the singularity problem of designing PID controllers in the integral and derivative plane and estimates achievable ranges of proportional gain of the PID controllers. A numerical cascaded ball and beam with unity feedback control of an SRV-DC motor and uncertain communication time delays in the system process demonstrate the application of this methodology. In this application, the additive weight bounds the additive errors for the cascaded ball and beam and the closed-loop SRV-DC motor system transfer function with the internal communication time delays.
\end{abstract}

Keywords: - Additive mixed sensitivity, uncertain time delays, PID controller, Ball and Beam.

Received: April 17, 2021. Revised: September 19, 2021. Accepted: October 25, 2021. Published: December 1, 2021.

\section{Introduction}

The proportional Integral Derivative (PID) controller is one of the most suitable controllers in many industrial systems. Although PID controllers have been functional in industrial systems for many years still there is a significant attempt in literature and industries to enhance the design of this type of controller. However, the robust and optimal tuning of the three parameters of PID controller coefficients is very challenging and time-consuming in many industrial applications. Furthermore, the time delay is one of the critical problems, particularly between the communication process of the hardware and software of a physical system. As a result, these problems become more of concern while the robustness and performance are also substantial for the practical applications with no information about the system dynamic. Therefore, the motivation for this paper is to enhance the design of a set of robust PID controller parameters for systems with uncertain time delays.

As the technology grows, the need to boost PID controller parameters becomes more critical since many industrial systems rely on this controller. Many researchers worldwide are writing more algorithms for designing the PID controller [1-16]. Verma and Kumar Padhy developed indirect internal modeling PID controller design for first and second-order system transfer functions [2]. This design applied to a canonical tank water level control the authors showed an improvement in time transient response
[2]. The system's stability was among the first constraints for tuning the PID controllers [3-4]. Sujoldžić and Watkins developed a PID controller to stabilize a system in the frequency domain [3]. Saeki defined a deterministic analysis to define the PID controller coefficient and developed a perdition for the number of unstable poles in the parameters space of the PID controller [4]. Sundaravadivua et al. reflected two degrees of freedom PID controller design to stabilize a first-order plus time delay models [5].

Ghousiya Begum et al. [8] applied an internal modeling controller with optimal $\mathrm{H}_{2}$ minimization to design a PID controller. They demonstrated this design to non-minimum phase integrating processes with time delays. Hussein Elmenfy tuned velocity PID-Fuzzy Power System Stabilizer controller gains by Particle Swarm Optimization technique [10]. Mustafa et al. [11] studied different control strategies for the liquid level in the two tanks system, such as classical PID controller and fuzzy adaptive PID controller for the mathematical model of the system. They demonstrated this study in MATLAB SIMULINK.

Wieneke and White [12] applied a pole placement method via PC running Lab-View in realtime. Senix sensors measured the ball position for the ball and beam feedback system. WU Yuanyuan et al. [13] designed a fuzzy PID controller for the ball's position in the ball and beam system and compared it 
with the traditional PID controller. Ford et al. [14] developed a Bode proportional derivative controller design for a ball position on a beam system. Carlos Bolívar-Vincenty et al. [15] applied both Newtonian and Lagrangian mechanics to derive the dynamical equations for the Ball and Beam system. They demonstrated that both methods provide identical results.

Different modelling of robustness of PID controller design has been the studied in the literatures [1], [6], [7],[9], [16], [17], [18], and [19]. In [16], the authors developed a PID controller algorithm for robust performance constraint single input single output systems with time delays. Gogoi et al. [17] designed a robust proportional-integral (PI) controller for a non-reheat steam generator unit. In [17], the authors did not look at the mixed sensitivity problem of the system. Jiménez-Cabas et al. [18] studied robust controllers such as $H_{\infty}$ algebraic Riccati equations, $H_{\infty}$ linear matrix inequalities techniques, and $\mathrm{H}_{2}$ controller design for a ball and beam system. They showed that $H_{\infty}$ offers better performance than the classical state variable feedback technique for the ball and beam system.

The current paper continues the previous techniques in [19] for the PID controller design. The contributions of this paper are in both theory and the application, which did not present in [19]. First, a set of algorithms in the present paper solves the singularity problem of designing PID controllers in the integral-derivative plane. Second, it introduces new modeling for the compensated ball and beam system. The objectives of the current paper are to augment the singularity procedures for the additive mixed sensitivity design of PID controller parameters for Single Input Single Output (SISO) with an uncertain time delay. The ball and beam system transfer function includes a cascading system with a closed-loop SRV-02 DC motor. The ball and beam modeling is an unstable double integrated transfer function [20], demonstrating this methodology's application.

The remainder of this paper presents as follows. First, the design technique; next, this method applies to a cascaded ball and beam with a closed-loop SRV02 DC motor transfer from Quanser Consulting, Incorporated to regulate the ball position on the beam. Finally, the conclusion of this paper is summarized.

\section{Design Technique}

The process plant $G_{p}$, with the perturbed plant $G_{\Delta}$, and the PID controller $G_{c}$, shapes a SISO system.
The reference input, the error signals, and the output are $R, Z$, and $Y$, respectively. The sensitivity function weight is $W_{P}$, the additive weight is $W_{A}$, and the uncertain perturbation is $\left|\Delta_{A}\right| \leq 1[21]$.

The process continuous-time system in the Laplace domain describes such as:

$$
G_{p}(s)=G_{o}(s) e^{-\tau s},
$$

where the nominal system dynamic transfer function is $G_{o}(s)$ and an uncertain communication time delay is $\tau$. The PID controller is:

$G_{c}(s)=K_{p}+\frac{K_{i}}{s}+K_{d} s$,

where the parameters proportional $K_{p}$, integral $K_{i}$, and derivative $K_{d}$, are the coefficients of the PID controller.

In frequency domain by substituting $s=j \omega$, the equivalent system transfer function in the equation (1) can be written based on its real and imaginary parts such as:

$G_{p}(j \omega)=R_{p}(\omega)+j I_{p}(\omega)$,

where the real numerical values of the data are $R_{p}(\omega)$ , and the imaginary numerical values of the data are $I_{p}(\omega)$. The PID controller in the equation (2) is written in the frequency domain such as:

$G_{c}(j \omega)=K_{p}-j \frac{K_{i}}{\omega}+j K_{d} \omega$.

The weights are complex functions and have a system dynamic. The additive weight is in the equation (5), and the sensitivity function weight is in the equation (6). These functions are defined in terms of their numerical real and imaginary data values such as:

$W_{A}(j \omega)=A_{A}(\omega)+j B_{A}(\omega)$,

$W_{P}(j \omega)=C_{P}(\omega)+j D_{P}(\omega)$.

In equations (5) and (6) the real numerical values of the data are $A_{A}(\omega), C_{P}(\omega)$, and the imaginary numerical values of the data are $B_{A}(\omega), D_{P}(\omega)$.

The fundamental goal is to find all achievable PID controllers that satisfy the additive mixed constraint 
of the system for all uncertain perturbations of $\left|\Delta_{A}(j \omega)\right| \leq 1[21]$. The additive mixed sensitivity constraint for the system is defined as [19]:

$\left(\left|W_{A}(\omega) G_{c}(\omega) S(\omega)\right|+\left|W_{P}(\omega) S(\omega)\right|\right) \leq \gamma \quad \forall \omega$,

In the equation, (7) the sensitivity function is $S(j \omega)=\frac{1}{1+G_{p}(j \omega) G_{c}(j \omega)}$ and $\gamma$ is a positive real number less than one that is the mixed sensitivity constraint. To find PID controller parameters, the complex function in the equation (7) is written in terms of their magnitudes and phase angles as:

$$
\left(\begin{array}{l}
|| W_{A}(j \omega) G_{c}(j \omega) S(j \omega)\left|e^{j\left(\angle W_{A}(j \omega) G_{c}(j \omega) S(j \omega)\right)}\right| \\
+ \\
|| W_{P}(j \omega) S(j \omega)\left|e^{j \angle W_{P}(j \omega) S(j \omega)}\right|
\end{array}\right) \leq \gamma, \forall \omega .
$$

Now for each value $\omega$, the equation (8) can be written as:

$$
\left(\begin{array}{l}
W_{A}(j \omega) G_{c}(j \omega) S(j \omega) e^{j \theta_{A}} \\
+ \\
W_{P}(j \omega) S(j \omega) e^{j \theta_{P}}
\end{array}\right) \leq \gamma, \forall \omega,
$$

where $\quad \theta_{A}=-\angle W_{A}(j \omega) G_{c}(j \omega) S(j \omega) \quad$ and $\theta_{P}=-\angle W_{P}(j \omega) S(j \omega)$ for some $\theta_{A} \in[0,2 \pi)$ and $\theta_{P} \in[0,2 \pi)$. Consequently, all PID controllers that satisfy equation (7) must lie at the intersection of all controllers that satisfy (9) for some $\theta_{A} \in[0,2 \pi)$ and $\theta_{P} \in[0,2 \pi)$. To accomplish this region, for each value of $\theta_{A} \in[0,2 \pi)$ and $\theta_{P} \in[0,2 \pi)$ all PID controllers on the boundary of the equation (9) are found [19].

It is easy to show from the equation (9) that PID controllers on this boundary must satisfy the following characteristic equation:

$P\left(\omega, \theta_{A}, \theta_{P}, \gamma\right)=0$,

where,

$$
\begin{aligned}
& P\left(\omega, \theta_{A}, \theta_{P}, \gamma\right)= \\
& 1+G_{p}(j \omega) G_{c}(j \omega)-\frac{1}{\gamma}\left(\begin{array}{l}
W_{A}(j \omega) G_{c}(j \omega) S(j \omega) e^{j \theta_{A}}+ \\
W_{P}(j \omega) S(j \omega) e^{j \theta_{P}}
\end{array}\right) .
\end{aligned}
$$

Note that equation (10) reduces to the frequency response of the standard closed-loop characteristic polynomial as $\gamma \rightarrow \infty$ [16]. Substituting equations (3) , (4), (5), (6), and $e^{j \theta_{A}}=\cos \theta_{A}+j \sin \theta_{A}$, and $e^{j \theta_{P}}=\cos \theta_{P}+j \sin \theta_{P}$ into the equation (10), the frequency response of this modified characteristic polynomial can be rewritten in terms of its real and imaginary functions such as:

$X_{R p}(\omega) K_{p}+X_{R i}(\omega) K_{i}+X_{R d}(\omega) K_{d}=Y_{R}(\omega)$,

and

$X_{I p}(\omega) K_{p}+X_{I i}(\omega) K_{i}+X_{I d}(\omega) K_{d}=Y_{I}(\omega)$,

where,

$$
\begin{aligned}
& X_{R p}(\omega)=\omega\left(R_{p}(\omega)-(\Phi(\omega))\right), \\
& X_{R i}(\omega)=\left(I_{p}(\omega)-(\Psi(\omega))\right), \\
& X_{R d}(\omega)=-\omega^{2}\left(I_{p}(\omega)-(\Psi(\omega))\right), \\
& Y_{R}(\omega)=\omega(-1+(\Omega(\omega))), \\
& X_{I p}(\omega)=\omega\left(I_{p}(\omega)-(\Psi(\omega))\right), \\
& X_{I i}(\omega)=\left(-R_{p}(\omega)+(\Phi(\omega))\right), \\
& X_{I d}(\omega)=-\omega^{2}\left(R_{p}(\omega)+(\Phi(\omega))\right), \\
& Y_{I}=\omega(\Lambda(\omega)),
\end{aligned}
$$

and,

$\Phi(\omega)=\frac{1}{\gamma}\left(A_{A}(\omega) \cos \theta_{A}-B_{A}(\omega) \sin \theta_{A}\right)$,

$\Psi(\omega)=\frac{1}{\gamma}\left(A_{A}(\omega) \sin \theta_{A}+B_{A}(\omega) \cos \theta_{A}\right)$,

$\Omega(\omega)=\frac{1}{\gamma}\left(C_{P}(\omega) \cos \theta_{P}-D_{P}(\omega) \sin \theta_{P}\right)$,

$\Lambda(\omega)=\frac{1}{\gamma}\left(C_{P}(\omega) \sin \theta_{P}+D_{P}(\omega) \cos \theta_{P}\right)$.

This is a three-dimensional system in terms of the controller parameters $K_{p}, K_{i}$, and $K_{d}$. In this paper, three methods are presented to find the set of PID controller parameters. The solutions are by fixing one coefficient and solving the equations (11) and (12) for the two other parameters. The solution of proportional-derivative $\left(K_{p}, K_{d}\right)$ and proportional-integral $\left(K_{p}, K_{i}\right)$ planes was presented in [19]. The solution of the integral-derivative $\left(K_{i}, K_{d}\right)$ plane did not present in the previous work.

\subsection{PID Controller Design in $\left(K_{p}, K_{d}\right)$ Plane}

After setting $K_{i}$ to a fixed value $\tilde{K}_{i}$, the equations (11) and (12) can be rewritten as: 


$$
\left[\begin{array}{cc}
X_{R p}(\omega) & X_{R d}(\omega) \\
X_{I p}(\omega) & X_{I d}(\omega)
\end{array}\right]\left[\begin{array}{c}
K_{p} \\
K_{d}
\end{array}\right]=\left[\begin{array}{c}
Y_{R}(\omega)-X_{R i}(\omega) \tilde{K}_{i} \\
Y_{I}(\omega)-X_{I i}(\omega) \tilde{K}_{i}
\end{array}\right] .
$$

Solving the equation (13) for all $\omega \neq 0, \theta_{A} \in[0,2 \pi$ ), and $\theta_{P} \in[0,2 \pi)$ gives the following expressions for the proportional and derivative parameters of PID controller:

$$
\begin{aligned}
& K_{p}\left(\omega, \theta_{A}, \theta_{P}, \gamma\right)= \\
& \frac{\left(\begin{array}{l}
-R_{p}(\omega)(1-\Omega(\omega))-I_{p}(\omega)(\Lambda(\omega))+(\Phi(\omega))+ \\
\left(\Upsilon_{1}(\omega) \cos \left(\theta_{P}-\theta_{A}\right)+\Upsilon_{2}(\omega) \sin \left(\theta_{P}-\theta_{A}\right)\right)
\end{array}\right)}{(\Theta(\omega))}, \\
& K_{d}\left(\omega, \theta_{A}, \theta_{P}, \gamma\right)=\frac{\tilde{K}_{i}}{\omega^{2}}+ \\
& \frac{\left(\begin{array}{l}
R_{p}(\omega)(\Lambda(\omega))-I_{p}(\omega)(-1+\Omega(\omega))-(\Psi(\omega))- \\
\left(\Upsilon_{1}(\omega) \sin \left(\theta_{P}-\theta_{A}\right)+\Upsilon_{2}(\omega) \cos \left(\theta_{P}-\theta_{A}\right)\right)
\end{array}\right)}{\omega(\Theta(\omega))},
\end{aligned}
$$

where,

$$
\begin{aligned}
\Upsilon_{1}(\omega) & =\frac{1}{\gamma^{2}}\left(A_{A}(\omega) C_{P}(\omega)+B_{A}(\omega) D_{P}(\omega)\right), \\
\Upsilon_{2}(\omega) & =\frac{1}{\gamma^{2}}\left(A_{A}(\omega) D_{P}(\omega)-B_{A}(\omega) C_{P}(\omega)\right), \\
\Theta(\omega)= & \left|G_{p}(j \omega)\right|^{2}-2\left(R_{p}(\omega)(\Phi(\omega))+I_{p}(\omega)(\Psi(\omega))\right)+ \\
& \frac{1}{\gamma^{2}}\left|W_{A}(j \omega)\right|^{2},
\end{aligned}
$$

and,

$\left|G_{p}(j \omega)\right|^{2}=R_{p}^{2}(\omega)+I_{p}^{2}(\omega)$,

$\left|W_{A}(j \omega)\right|^{2}=A_{A}^{2}(\omega)+B_{A}^{2}(\omega)$.

\subsection{PID Controller Design in $\left(K_{i}, K_{d}\right)$ Plane}

One of the contributions of the current paper is to find the mixed sensitivity and stability boundaries of the equation (10) in the $\left(K_{i}, K_{d}\right)$ plane for a fixed value of $K_{p}$. Setting $K_{p}=\tilde{K}_{p}$, the equations (11) and (12) can be rewritten as:

$\left[\begin{array}{cc}X_{R i} & X_{R d} \\ X_{I i} & X_{I d}\end{array}\right]\left[\begin{array}{c}K_{i} \\ K_{d}\end{array}\right]=\left[\begin{array}{c}Y_{R}-X_{R p} \tilde{K}_{p} \\ Y_{I}-X_{I p} \tilde{K}_{p}\end{array}\right]$.

The solution of (16) for all $\omega \in(0, \infty)$ creates a singularity problem. There are two steps to solve this problem. First, the range of $K_{p}=\tilde{K}_{p}$ is determined in the ( $\left.K_{p}, \omega\right)$ plane from the equation (14). The plot of proportional gains in the equation (14) versus the frequency in the first quarter gives the scope of prediction for all achievable proportional gains. The second part of this solution is to determine the specific frequencies, such as $\omega_{i}$. These frequencies are the interception of $K_{p}(\omega)$ from the equation (14) and the frequency range of $\omega$. Note that the stability boundaries can be selected from the equation (14) as $\gamma \rightarrow \infty$ in the first quarter. As a result, the solutions for $K_{d}\left(\omega_{i}\right)$ and $K_{i}\left(\omega_{i}\right)$ parameters in the equation (16) must satisfy either of the following straight-line equations:

$$
\begin{aligned}
& K_{d}\left(\omega_{i}, \theta_{A}, \theta_{P}, \gamma\right)=\frac{\tilde{K}_{i}}{\omega_{i}{ }^{2}}+ \\
& \frac{\left(\begin{array}{l}
R_{p}\left(\omega_{i}\right)\left(\Lambda\left(\omega_{i}\right)\right)-I_{p}\left(\omega_{i}\right)\left(-1+\Omega\left(\omega_{i}\right)\right)-\left(\Psi\left(\omega_{i}\right)\right)- \\
\left(\Upsilon_{1}\left(\omega_{i}\right) \sin \left(\theta_{P}-\theta_{A}\right)+\Upsilon_{2}\left(\omega_{i}\right) \cos \left(\theta_{P}-\theta_{A}\right)\right)
\end{array}\right)}{\omega_{i}\left(\Theta\left(\omega_{i}\right)\right)},
\end{aligned}
$$

$$
\begin{aligned}
& K_{i}\left(\omega_{i}, \theta_{A}, \theta_{P}, \gamma\right)=\omega_{i}^{2} \tilde{K}_{d}+ \\
& \frac{\omega_{i}\left(\begin{array}{l}
-R_{p}\left(\omega_{i}\right)\left(\Lambda\left(\omega_{i}\right)\right)+I_{p}\left(\omega_{i}\right)\left(-1+\Omega\left(\omega_{i}\right)\right)+\Psi\left(\omega_{i}\right)+ \\
\left(\Upsilon_{1}\left(\omega_{i}\right) \sin \left(\theta_{P}-\theta_{A}\right)+\Upsilon_{2}\left(\omega_{i}\right) \cos \left(\theta_{P}-\theta_{A}\right)\right)
\end{array}\right)}{\left(\Theta\left(\omega_{i}\right)\right)} .
\end{aligned}
$$

\subsection{PID Controller Design in $\left(K_{p}, K_{i}\right)$ Plane}

The additive mixed sensitivity and stability boundaries of the equation (10) are found in the $\left(K_{p}, K_{i}\right)$ plane for a fixed value of $K_{d}$. After setting $K_{d}$ to the fixed value $\tilde{K}_{d}$, the equations (11) and (12) can be rewritten as:

$$
\left[\begin{array}{cc}
X_{R p}(\omega) & X_{R i}(\omega) \\
X_{I p}(\omega) & X_{I i}(\omega)
\end{array}\right]\left[\begin{array}{c}
K_{p} \\
K_{i}
\end{array}\right]=\left[\begin{array}{c}
Y_{R}(\omega)-X_{R d}(\omega) \tilde{K}_{d} \\
Y_{I}(\omega)-X_{I d}(\omega) \tilde{K}_{d}
\end{array}\right]
$$

Solving the equation (19) for all $\omega \neq 0$, $\theta_{A} \in[0,2 \pi)$ and $\theta_{P} \in[0,2 \pi)$ gives the same expression as the equation (14) for $K_{p}\left(\omega, \theta_{A}, \theta_{P}, \gamma\right)$, and the following equation for the integral parameter of PID controllers: 


$$
\begin{aligned}
& K_{i}\left(\omega, \theta_{A}, \theta_{P}, \gamma\right)=\omega^{2} \tilde{K}_{d}+ \\
& \frac{\omega\left(\begin{array}{l}
-R_{p}(\omega)(\Lambda(\omega))+I_{p}(\omega)(-1+\Omega(\omega))+\Psi(\omega)+ \\
\left(\Upsilon_{1}(\omega) \sin \left(\theta_{P}-\theta_{A}\right)+\Upsilon_{2}(\omega) \cos \left(\theta_{P}-\theta_{A}\right)\right)
\end{array}\right)}{(\Theta(\omega))} .
\end{aligned}
$$

\section{Numerical Example}

In this section, PID controllers are designed to demonstrate the formulas from the additive mixed sensitivity constraint. A cascaded ball and beam and an SRV-DC are shown in Figure 1. Therefore, to clarify the design process, the design goal and the design discussion and results in both $\left(K_{i}, K_{d}\right)$ and $\left(K_{p}, K_{d}\right)$ planes are presented in this section.

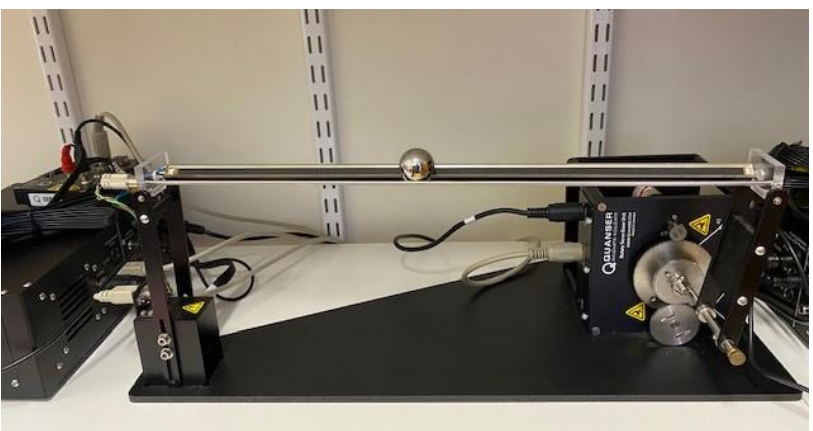

Fig. 1. Ball and beam system

\subsection{Design Goals:}

The main goal is to find all PID controllers that stabilize a ball position on a beam and satisfy the additive mixed sensitivity constraint in the equation (7). In addition, the closed-loop step response requires an overshoot of less than $25 \%$ and a settling time of fewer than 12 seconds for some of the PID controllers in the mixed sensitivity region.

The nominal SRV-DC motor in equation (21) and the ball and beam system in equation (22) transfer functions are [20]:

$$
\begin{gathered}
G_{m o l}=\frac{60.24}{s^{2}+39.37 s}, \\
G_{b b}(s)=\frac{0.419}{s^{2}} .
\end{gathered}
$$

The block diagram of the cascaded ball and beam with unity feedback control of the SRV-DC motor is shown in Figure 2. The closed-loop transfer function of the SRV-DC motor is:

$$
G_{m}=\frac{60.24}{s^{2}+39.37 s+60.24} .
$$

The system dynamic transfer function of the cascaded ball and beam and the closed-loop DC motor is:

$$
\begin{aligned}
& G_{o}(s)=G_{m}(s) G_{b b}(s), \\
& G_{o}(s)=\frac{25.24}{s^{4}+39.37 s^{3}+60.24 s^{2}} .
\end{aligned}
$$

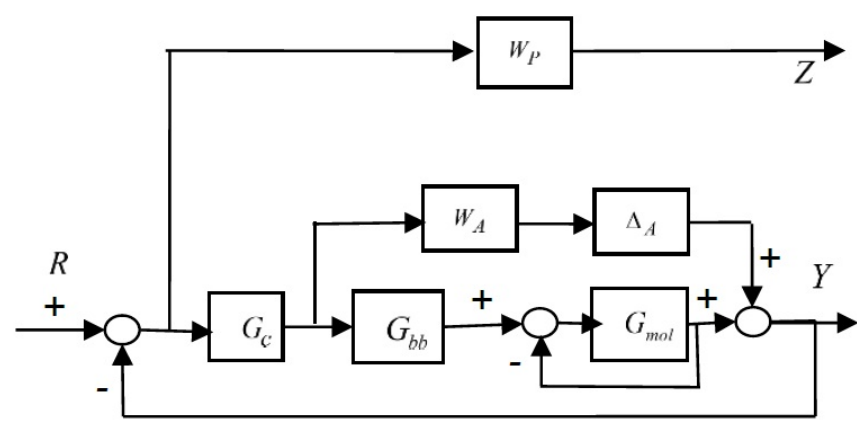

Fig. 2. Block diagram of the cascaded ball and beam with SRV-DC motor

The assumption here is that an uncertain communication time delay between $0<\tau<0.3$ seconds occurs in the system process. Therefore, a communication time delay of 0.2 seconds in this interval is chosen to demonstrate the design process. The nominal model of the system in the equation (3) is:

$$
G_{p}(s)=\frac{25.24}{s^{4}+39.37 s^{3}+60.24 s^{2}} e^{-\tau s} .
$$

\subsection{Design Results and Discussion:}

The additive weight is designed to bound the additive errors [21] for the system transfer function. The ball and beam system in equation (25) with all the interval of communication time delays between $0<\tau<0.3$ is shown in Figure 3. The additive weight transfer function is designed as shown in the bold-blue-color in magnitude frequency response for the cascaded ball and beam and SRV-02 DC motor system in Figure 3 such as equation (26):

$$
W_{A}(s)=\frac{93.75}{s^{4}+51 s^{3}+675.1 s^{2}+626 s+0.9375} .
$$

The sensitivity weight function is chosen to satisfy the performance requirements of the percent overshoot and settling time for the closed-loop 
system. Therefore, the sensitivity weight is designed such as:

$$
W_{P}(s)=\frac{0.78 s+1.37}{s^{2}+4.75 s+4.63}
$$

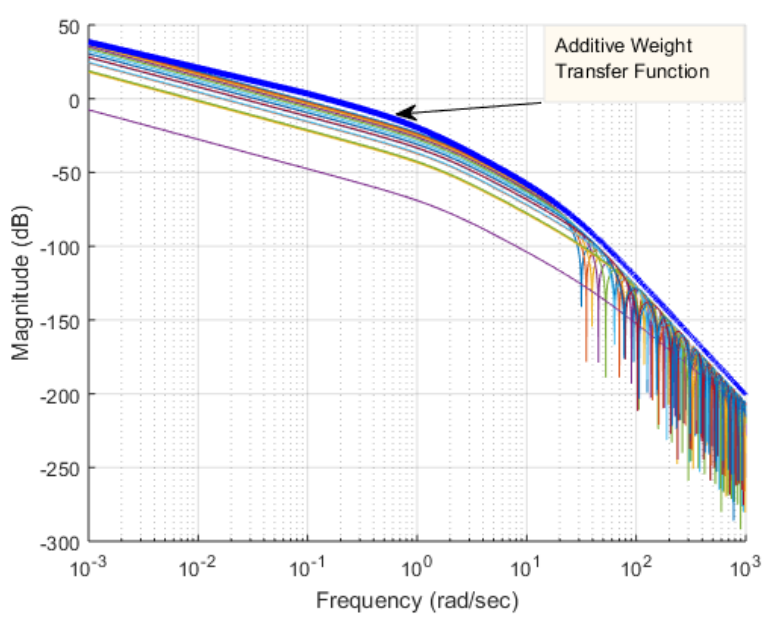

Fig. 3. The additive weight (bold blue color) is designed to bound the additive uncertainty errors

The plot of proportional gain in the equation (14) and various frequency ranges shows in Figure 4. This Figure shows proportional gains of PID controllers $\left(K_{p} \in(0,3.65)\right)$ for the stability boundary in red and mixed sensitivity in light green. For each curve in Figure 4, the $\omega_{i}{ }^{\prime} s$ are the frequencies at which $K_{p}(\omega)=\tilde{K}_{p}=0.1$. Each $\omega_{i}$ is substituted (17) or (18) in the $\left(K_{i}, K_{d}\right)$ plane to find the required region for choosing the integral and derivative PID controller parameters. Also, there is a boundary at $K_{i}(0)=0$.

The PID stability boundary of the nominal system can be found by setting $\gamma=\infty$ in the equations (17) or (18). The nominal stability boundary is shown in the red line. The area that satisfies the mixed sensitivity is the dotted-blue area. In this plane, the PID controller is designed to meet the mixed sensitivity constraint in (7) by selecting the upper limit constraint of additive sensitivity constraint $\gamma=1$ in the equations (17) or (18) for some $\theta_{A} \in[0,2 \pi), \theta_{P} \in[0,2 \pi)$, and finding the intersection of all regions. The area that met the additive mixed sensitivity constraint and the nominal stability boundary are shown in Figure 5.

To verify the results, an arbitrary PID controller from this region is chosen, such as:

$G_{c}(s)=0.1+\frac{0.005}{s}+1.937 s$
Substituting equations (25), (26), (27), and (28), into the equation (7) gives the additive mixed sensitivity function magnitude of $\left(\left|W_{A}(s) G_{c}(s) S(s)\right|+\left|W_{P}(s) S(s)\right|\right)=0.696$ as it shows in Figure 6. Therefore, as the magnitude of the mixed sensitivity system is less than one, the design goal for the mixed sensitivity constraint has been met.

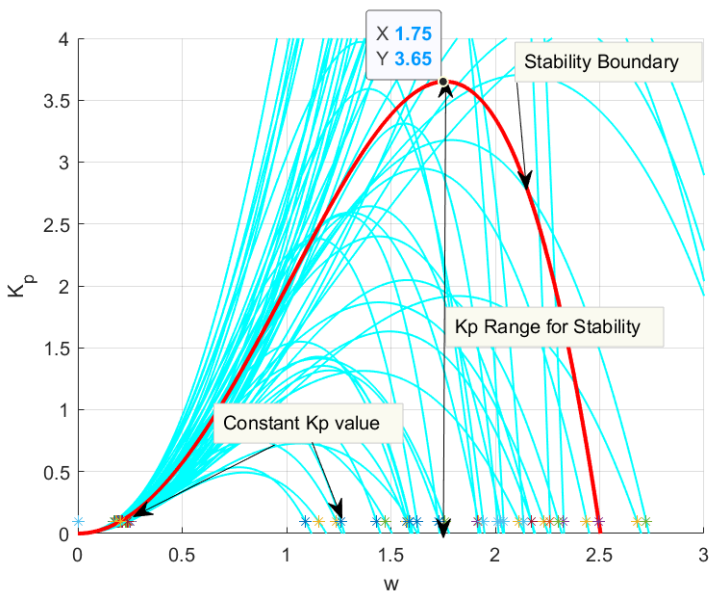

Fig. 4. The plot of proportional gain versus frequency

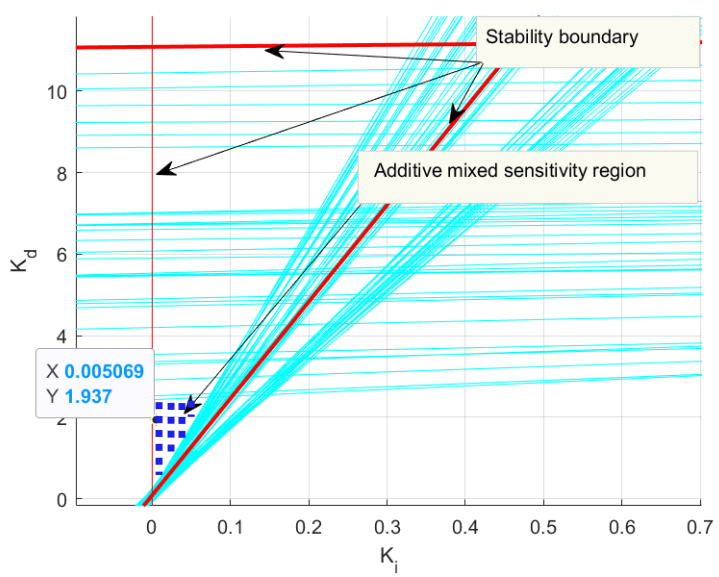

Fig. 5. Stability boundary and additive mixed sensitivity region in the $\left(K_{i}, K_{d}\right)$ plane

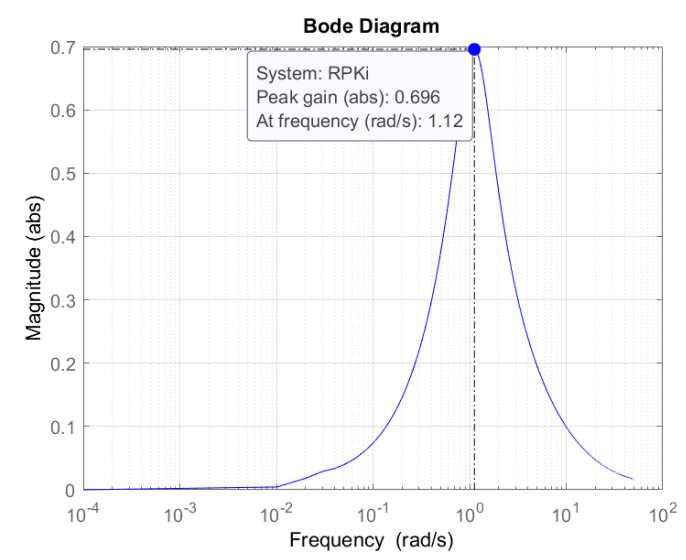

Fig. 6. The magnitude frequency response of the compensated system with PID controller in (28) 
The second method uses equations (14) and (15) is applied in the $\left(K_{p}, K_{d}\right)$ plane for a fixed value of $K_{i}=\tilde{K}_{i}$. The PID stability boundary of the system can be found by setting $\gamma=\infty$ in equations (14) and (15) the $\left(K_{p}, K_{d}\right)$ plane. All PID controllers that satisfy the mixed sensitivity constraint in the equation (7) are found by selecting $\gamma=1$ in equations (14) and (15) for some $\theta_{A} \in[0,2 \pi)$ and $\theta_{P} \in[0,2 \pi)$, and then finding the intersection of all regions in the $\left(K_{p}, K_{d}\right)$ plane.

This method demonstrates in the $\left(K_{p}, K_{d}\right)$ plane for a fixed value of $\tilde{K}_{i}=0.001$. The region that satisfies the mixed sensitivity constraint and the nominal stability boundary is shown in Figure 7 . The nominal stability boundary is shown in the red line. The intersection of all regions inside the nominal stability boundary of the $\left(K_{p}, K_{d}\right)$ plane is shown inside the stability boundary with dotted-blue lines in Figure 7. Thus, All of the selected PID controllers in the intersection area satisfy the additive mixed sensitivity constraint in the equation (7).

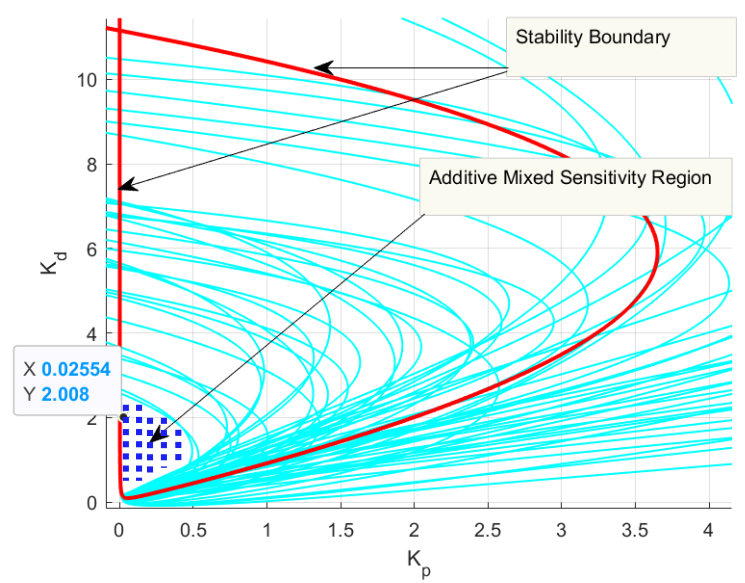

Fig. 7. Stability boundary and additive mixed sensitivity region in the $\left(K_{p}, K_{d}\right)$ plane

To verify the results, an arbitrary controller from this region is chosen that gives the PID controller such as:

$G_{c}(s)=0.025+\frac{0.001}{s}+2.008 s$.

Substituting equations (26), (27), and (29), into the equation gives $\left(\left|W_{A}(s) G_{c}(s) S(s)\right|+\left|W_{P}(s) S(s)\right|\right)=0.696$, as shown in Figure 8 , the magnitude of the frequency response of the mixed sensitivity function is less than one the design goal is met.

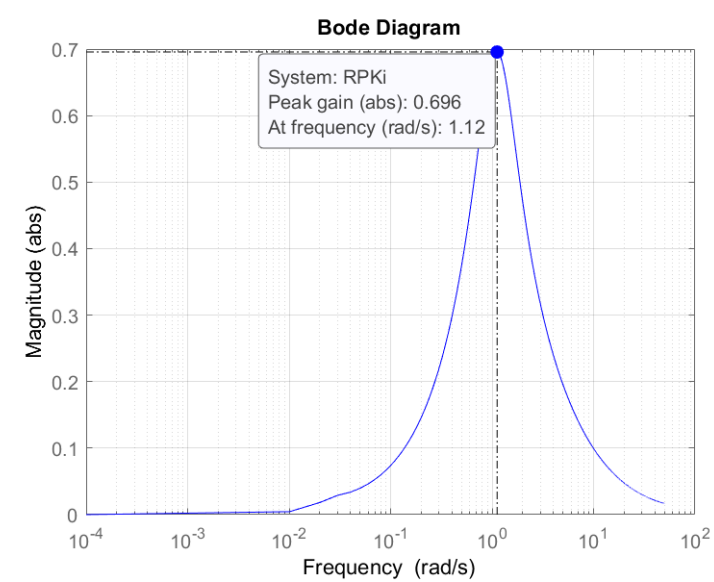

Fig. 8. The magnitude frequency response of the compensated system with PID controller in (29)

The step responses of the closed-loop system with the PID controller in the equation (29) and various time delays in the interval of $(0.005,0.3)$ seconds are shown in Figure 9. As can be seen, the closed-loop step responses all have an overshoot of less than $25 \%$ and a settling time of fewer than 12 seconds. Furthermore, the maximum setting time is 6.15 seconds, and the maximum percent overshoot is $14.7 \%$. All the closed-loop time responses have a steady-state error of zero. Thus, all the closed-loop step response performances have met the expectations.

The results for $\left(K_{p}, K_{i}\right)$ plane for a fixed value of $K_{d}=\tilde{K}_{d}$ is similar to the $\left(K_{p}, K_{d}\right)$ plane, but it applies the equations (14) and (20) in $\left(K_{p}, K_{d}\right)$ plane for some $\theta_{A} \in[0,2 \pi)$ and $\theta_{P} \in[0,2 \pi)$, then finding the intersection of all regions. These results correspond to the PID controller parameters for the proportional and integral gains and fixed value of derivative gain.

The research shows the flexibility of designers for enhancing PID controller coefficients by minimizing the area of PID controller selections. Each methodology of designing the PID controller described in this paper meets the additive mixed sensitivity constraints to make the robust controller develop. In each plane, there is at least a PID controller that meets all the time domain specifications due to the uncertain delays in the nominal system. 


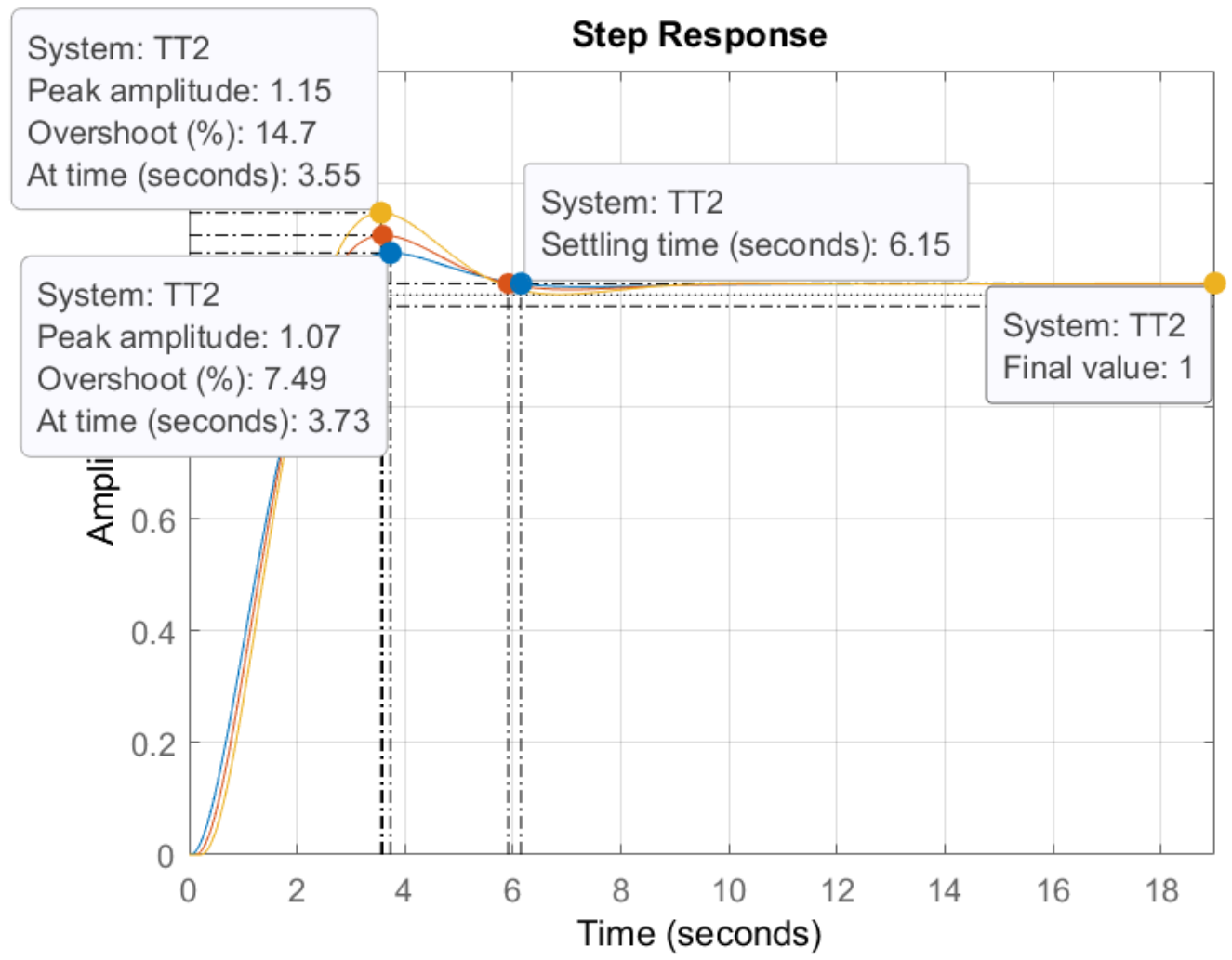

Fig. 9. The closed-loop step responses with PID controller in (29).

\section{Conclusion}

This paper presented an algorithm for singularity solution of Proportional Integral Derivative (PID) controllers that stabilized a continuous-time system and satisfied additive mixed sensitivity constraint with an uncertain time delay. This algorithm estimated all achievable ranges of proportional gains and solved the singularity problem of designing PID controllers in the integral and derivative plane. A numerical cascaded ball and beam and closed-loop SRV-DC motor system transfer function with uncertain communication time delays in the system process demonstrated the application of this methodology. In this application, the additive weight was designed to bound the additive errors for the cascaded ball and beam and the SRV-DC motor system closed-loop system transfer function with an internal communication time delay. Future work is to continue this methodology to Smith's predictor modeling of the system.

\section{References}

[1] S. P. Bhattacharyya, A. Datta, and H., Keel, Linear Control Theory Structure, Robustness, and Optimization. CRC Press, Taylor \&Francis Group, Boca Raton, FL, 2009.
[2] B. Verma1, P. Kumar Padhy, Indirect IMCPID Controller Design, IET Control Theory \& Applications., Vol. 13 Iss. 2, 2018, pp. 297305.

[3] S. Sujoldžić and J. M. Watkins, Stabilization of an Arbitrary Order Transfer Function with Time Delay Using PID Controller," Proceedings of $45^{\text {th }}$ IEEE Conference on Decision and Control, San Diego, CA, 2006.

[4] M. Saeki, Properties of Stabilizing PID Gain Set in Parameter Space, IEEE Transactions on Automatic Control, Vol. 52, No. 9, 2007, pp. 1710-1715.

[5] K. Sundaravadivua, S. Sivakumara , N. Hariprasada, 2DOF PID Controller Design for a Class of FOPTD Models an Analysis with Heuristic Algorithms, Procedia Computer Science 48, 2015, pp. $90-95$.

[6] Z. Wu, D. Li, and Y. Xue, A New PID Controller Design with Constraints on Relative Delay Margin for First Order Plus Dead-Time Systems, Processes, 7, 2019, 713.

[7] M. Armin, P. Nath Roy, S. K. Sarkar, S. K. Das, LMI-Based Robust PID Controller Design for Voltage Control of Islanded Microgrid, Chinese Automatic Control Society and John Wiley \& Sons Australia, Ltd, Asian 
Journal of Control, Vol. 20, No. 5, 2018, pp. 2014-2025.

[8] K. Ghousiya Begum, A. Seshagiri Rao, T. K. Radhakrishnan, Enhanced IMC Based PID Controller Design for Non-Minimum Phase (NMP) Integrating Processes with Time Delays, ISA Transactions, Vol. 68, 2017, pp. 223-234.

[9] M. Saeki, "Fixed structure PID controller design for standard $H_{\infty}$ control problem," Automatica, Vol. 42, 2006, pp. 93-100.

[10] T. Hussein Elmenfy, Design of Velocity PIDFuzzy Power System Stabilizer Using Particle Swarm Optimization, WSEAS Transactions on Systems, Vol. 20, 2021, pp. 9-14.

[11] K. Mustafa, A. Albagoul, M. Saad, "Design of Fuzzy Adaptive PID Controller for Nonlinear Multivariable Process," WSEAS Transactions on Systems, Vol. 18, 2019, pp. 262-269.

[12] J. Wieneke and W. N. White, A Feasibility Assessment of Using Ultrasonic Sensor Position Feedback for a Ball-and-Beam Apparatus, Proceedings of the 2011 American Control Conference, San Francisco, California.

[13] WU Yuanyuan, LIU Yongxin, Fuzzy PID Controller Design and Implement in BallBeam System, Proceedings of the 34th Chinese Control Conference, 2015, pp. 36133616.

[14] J. K. Ford and T. Emami, "Implementation of a Ball and Beam Control System Using PD Bode Design," International Journal of Modern Engineering, Vol. 18, No. 2, 2018.

[15] G. Carlos Bolívar-Vincenty and G. Beauchamp-Báez, Modeling the Ball-andBeam System from Newtonian Mechanics and from Lagrange Methods, Proceedings of the $12^{\text {th }}$ Latin American and Caribbean Conference for Engineering and Technology, July 2014.

[16] T. Emami and J. M. Watkins, Robust performance characterization of PID controllers in the frequency domain, WSEAS Trans. on Systems and Control, vol. 4, no. 5, 2009, pp. 232-242.

[17] M. Gogoi, T. Emami, and J. M. Watkins, Robust Stability Design of PI Controllers for a Non-Reheat Steam Generator Unit, Proceedings of the 2010 ASME Dynamic Systems and Control Conference," Cambridge, Massachusetts.

[18] J. Jiménez-Cabas, F. Meléndez-Pertuz, Robust Control of the Classic Dynamic Ball and Beam System, Springer Nature Switzerland $A G$
2020, ICCSA 2020, LNCS 12251, pp. 134144, 2020.

[19] T. Emami, Mixed Sensitivity Design of PID Controller- Applied to a Ball and Beam System, Proceedings of the 2017 ASME International Mechanical Engineering Congress and Exposition (IMECE), Tampa, Florida.

[20] J. Apkarian, P. Karam, M. Lévis, and H. Gurocak Ball and Beam Experiment for MATLAB $^{\circledR} /$ Simulink $^{\circledR} \quad$ Users, Quanser Innovate.

[21] S. Skogestad and I. Postlethwaite, Multivariable Feedback Control Analysis and Design. John Wiley, 2007.

\section{Creative Commons Attribution License 4.0 (Attribution 4.0 International, CC BY 4.0)}

This article is published under the terms of the Creative Commons Attribution License 4.0 https://creativecommons.org/licenses/by/4.0/deed.en_US 